\title{
NONLINEAR ANALYSIS OF AIRFOIL HIGH-INTENSITY GUST RESPONSE USING A HIGH-ORDER PREFACTORED COMPACT CODE
}

\author{
A. Crivellini* \\ Universita Degli Studi Di Ancona, Italy \\ V. Golubev ${ }^{\dagger}$, R. Mankbadi ${ }^{\ddagger}$ \\ Embry-Riddle Aeronautical University \\ J. Scott ${ }^{\S}$, R. Hixon ${ }^{\mathbb{I}}$, L. Povinelli ${ }^{\#}$ \\ NASA Glenn Research Center
}

\begin{abstract}
The nonlinear response of symmetric and loaded airfoils to an impinging vortical gust is investigated in the parametric space of gust dimension, intensity, and frequency. The study, which was designed to investigate the validity limits for a linear analysis, is implemented by applying a nonlinear high-order prefactored compact code and comparing results with linear solutions from the GUST3D frequency-domain solver. Both the unsteady aerodynamic and acoustic gust responses are examined.
\end{abstract}

\section{INTRODUCTION}

Most unsteady aerodynamic and aeroacoustic analyses considering interaction of upstream flow nonuniformities with downstream structural components follow linear theory wherein the unsteady part of the flow is considered a small perturbation about the steady mean flowfield. This approach, which relies on solutions to the linearized Euler equations, is adopted, for example, in problems dealing with rotorstator interactions where the rotor wakes are represented as low-intensity flow disturbances (vortical gusts) convected by the mean flow. This paper examines the limits of the linear theory when the unsteady vortical flow velocity component is, in fact, not too small compared to the steady mean flow velocity. Thus, the interaction problem is investigated by imposing a finite-amplitude gust on the mean flow, and a nonlinear flow

*Research Associate

$\dagger$ Assistant Professor, Member of AIAA.

$\ddagger$ Professor, Associate Fellow of AIAA

$\S$ Senior Research Scientist, Senior Member of AIAA

ISenior Research Associate, Member of AIAA

\#Chief Scientist, Fellow of AIAA analysis is then applied to examine possible deviations from the linear theory predictions. The problem of gustairfoil interaction is selected for this study as it presents a widely used benchmark in unsteady aerodynamics.

To the authors' best knowledge, no detailed, systematic study of high-intensity - gust response has been conducted so far (at least for realistic airfoil profiles), although nonlinear Euler and Navier-Stokes solvers have been in use for unsteady problems in turbomachinery for at least a decade (see [1] for a good review of the subject). A fully nonlinear viscous study of gust-airfoil interaction has been previously conducted by Lockard and Morris [2] with assumption of a small-amplitude perturbation. Hence, most observed nonlinear effects in [2] were related to gusttriggered instabilities in the viscous wake. In fact, in the present study, a similar mechanism giving rise to inviscid wake instability remains a crucial factor in describing the nonlinear response effects. In general, such effects to be investigated may include (e.g., [3], pp.863-873) generation of higher harmonics (both for single and multiple frequency input) and combination tones (for multiple-frequency input) resulting from nonlinear self-interaction and exchange of energy between multiple imposed frequencies. In certain cases, the effects of nonlinear interaction between the imposed harmonic(s) and the mean flow, including exchange of energy, can also be present. It is expected that these effects combined should become increasingly more noticeable at higher gust intensities when they would thus produce a more significant impact on the unsteady airfoil response, particularly on the unsteady aerodynamic loading and the acoustic directivity pattern.

The present work extends the analysis of the gust-airfoil interaction problem first presented in [4] and [5]. Results of the nonlinear inviscid analysis based on explicit time marching with prefactored sixth-order

American Institute of Aeronautics and Astronautics

This is a preprint or reprint of a paper intended for presentation at a conference. Because changes may be made before formal 
compact scheme for spatial differences, are compared with solutions from a frequency-domain linearized Euler solver GUST3D [6]. The unsteady response to one-dimensional and two-dimensional gusts is examined for both loaded and unloaded thick Joukowski airfoils in a subsonic flow.

\section{FORMULATION}

The analysis of nonlinear inviscid gust-airfoil interaction is based on the numerical solution to the nonlinear Euler equations which can be written in twodimensional Cartesian coordinates as

$$
\frac{\partial Q}{\partial t}+\frac{\partial E}{\partial x}+\frac{\partial F}{\partial y}=0
$$

where $Q$ is the solution vector, and $F$ and $G$ are the flux vectors:

$$
\begin{aligned}
& Q=\left[\begin{array}{c}
\rho \\
\rho u \\
\rho v \\
E
\end{array}\right], \\
& E=\left[\begin{array}{c}
\rho u \\
\rho u^{2}+p \\
\rho u v \\
u(E+p)
\end{array}\right], \\
& F=\left[\begin{array}{c}
\rho v \\
\rho u v \\
\rho v^{2}+p \\
v(E+p)
\end{array}\right],
\end{aligned}
$$

and

$$
p=(\gamma-1)\left(E-\frac{1}{2} \rho\left(u^{2}+v^{2}\right)\right)
$$

Since the gust response is investigated for the complexshaped Joukowksi airfoil, it requires recasting the equations in generalized curvilinear coordinates, with the chain-rule curvilinear Euler equations written as

$$
\frac{\partial Q}{\partial t}+\frac{\partial \xi}{\partial x} \frac{\partial E}{\partial \xi}+\frac{\partial \eta}{\partial x} \frac{\partial E}{\partial \eta}+\frac{\partial \xi}{\partial y} \frac{\partial F}{\partial \xi}+\frac{\partial \eta}{\partial y} \frac{\partial F}{\partial \eta}=0
$$

The numerical formulation established in [4] and [5] uses the low-storage $4^{\text {th }}$ order 5-6 Low Dispersion and Dissipation Runge-Kutta scheme [7] for time marching, and the prefactored $6^{\text {th }}$ order compact scheme and explicit boundary stencils for spatial derivatives [8]. A $10^{\text {th }}$ order explicit filter is used at every stage of the Runge-Kutta solver to provide dissipation.

For the analysis of the gust response problem, a C-grid 2D topology is used for the $12 \%$ thick Joukowski airfoil, with the grid extending at least 10 chords away from the surface in each direction. All the meshes are generated as single blocks by the GridPro commercial software [9]. More details on the numerical implementation are included below.

\section{GUST AND AIRFOIL CONFIGURATIONS AND FLOW INITIALIZATION}

In the current work, the nonlinear gust response is examined for a series of imposed gust intensities and frequencies. Each vortical gust harmonic is initially imposed on the mean flow with the following distribution:

$$
\begin{aligned}
& u_{\text {gust }}=-\left(\frac{\varepsilon \beta M}{\sqrt{\alpha^{2}+\beta^{2}}}\right) \cos (\alpha x+\beta y-\omega t) \\
& v_{\text {gust }}=-\left(\frac{\varepsilon \alpha M}{\sqrt{\alpha^{2}+\beta^{2}}}\right) \cos (\alpha x+\beta y-\omega t)
\end{aligned}
$$

where $\varepsilon$ is the gust intensity relative to the mean flow, $\alpha$ and $\beta$ are the gust wave numbers in the $x$ and $y$ directions, and $\omega$ is the imposed gust frequency. The mean flow is defined far upstream from the airfoil as:

$$
\begin{aligned}
& \bar{\rho}=1 \\
& \bar{u}=M \\
& \bar{v}=0 \\
& \bar{p}=\frac{1}{\gamma}\left(\bar{\rho}^{\gamma}\right)
\end{aligned}
$$

where $M$ is the upstream mean flow Mach number, and $\gamma=1.4$. For this paper's computations, the Mach number is fixed at $M=0.5$.

The flow is initialized throughout the physical domain, with the gust superimposed on the mean flow. In addition, at the inflow boundary, the acoustic radiation condition given by Tam and Webb [10] is applied to the outgoing perturbation which is defined, e.g., for $u$ component as $u_{B C}=u_{\text {boundary }}-\bar{u}-u_{\text {gust }}$. 
The following two configurations for the airfoil geometry are considered:

(A1) Unloaded symmetric $12 \%$-thick Joukowski airfoil (no angle of attack);

(A2) Loaded 12\%-thick 2\%-cambered Joukowski airfoil at a two-degree angle of attack.

Two configurations for the impinging gust are examined:

(G1) One-dimensional (1D) transverse gust with $\alpha=2 k, \beta=0, \omega=2 k M$ in (5);

(G2) Two-dimensional (2D) gust with $\alpha=2 k, \beta=2 k, \omega=2 k M$ in (5),

where $k$ is the reduced frequency of the gust nondimensionalized by the half-chord and the upstream flow velocity: In the computations, both the gust intensity and reduced frequency are varied.

\section{RESULTS AND DISCUSSION}

The focus of this study is on nonlinear effects of highintensity gust interaction with an aiffoil. Hence, it is important to provide comparison for different gust intensity levels, with the corresponding low-intensity results assumed to fall within the linear response limits. At first, the results of the numerical analysis for all gust and airfoil configurations are validated against the GUST3D linear analysis calculations. Following this, a systematic study of gust intensity effects on various nonlinear response aspects is carried out, with the most important findings discussed and illustrated below.

\section{Numerical Implementation}

The computational code performing nonlinear unsteady analysis is written in Fortran 90 and uses the MPI library for parallelization. Numerical solutions are obtained through parallel implementation on the Beowulf-type Linux Athlon cluster consisting of eight dual-processor $1.4 \mathrm{GHz}$ AMD compute nodes, each with $1 G B$ of PC2100DDR memory. The nodes are connected by five full duplex fast ethernet connections to the other nodes in the cluster using a symmetric interconnection scheme. The MPICH implementation of MPI is used [11].

The parallel performance of the code is shown in Figure 1 , with the noticeable decrease in the computational efficiency observed when more then eight processors are involved in the computations. This is due to the fact that the cluster starts to utilize both processors of the single nodes sharing the same bus allocation on the motherboard. For instance, the same computation performed with two processors from different nodes is observed to have the parallel efficiency of $95 \%$, compared to $73 \%$ when two processors from the same node are used. This trend is typical of communicationintensive numerical implementations.

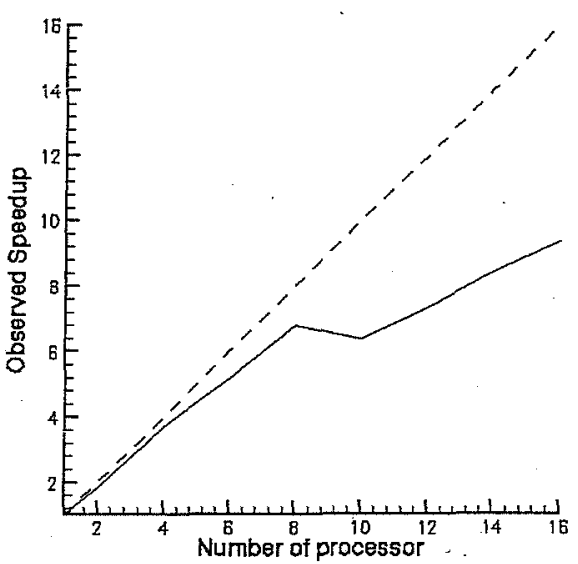

Figure 1: Observed speedup for parallel runs.

In this work, the simulations are run for longer time periods compared to [4] since stable, time-accurate solutions are sought not only for imposed gust frequencies but also for higher harmonics and combination tones. In terms of the non-dimensional time (normalized by the airfoil half-chord and the speed of sound), the values between 360 and 450 are found necessary for the more slowly converging loaded airfoil simulations to accurately resolve the mean flowfield and the acoustic field. More time iterations are required for lower gust frequency cases in order to march through an adequate number of longer periods. Some small differences in results compared to the similar cases in [4] can be attributed to longer convergence histories in the present runs. Finally, to quantify the computational effort, a 16-hour numerical run is required to march to the non-dimensional time value of 360 , which corresponds to 70,000 iterations on the $600 \times 240$ grid using all sixteen processors.

\section{Validation}

The validation of the nonlinear code results with the linear solutions from the GUST3D solver has been previously accomplished in . [4], where thorough grid density and domain size sensitivity studies have also been conducted. The reader is referred to this paper for more details. In the present work, this validation is further extended to numerical simulations on meshes with grid densities $600 \times 240$ (for the cambered airfoil cases) and $591 \times 238$ (for the symmetric airfoil cases). Similar to [4], the grids are generated by GridPro using algebraic clustering around the profile in the normal direction $(\Delta n=0.01)$ and near the trailing edge 
$(\Delta x=0.01)$, with the stretching ratio of 1.05 used to expand to the far field. Note that some oscillations and loss of accuracy are found near the trailing edge, which is due to the singularity treatment in the grid topology. Nevertheless, a careful clustering of grid points near the trailing edge may help to keep these effects localized, with minimal impact on the overall solution.

The results are presented for two reduced frequencies of the gust, $\mathrm{k}=0.1$ in Figures 2 and 3 , and $\mathrm{k}=1.0$ in Figures 4 and 5. For the selected airfoil and gust configurations (A1-G1), (A1-G2), (A2-G1), (A2-G2), Figures 2 and 4 show the acoustic intensity of the unsteady response at a distance of one chord length away from the centerpoint of the airfoil, while Figures 3 and 5 show results for the same quantity at a distance of four chord lengths. For each case, three results are compared including the low-intensity gust response (for $\varepsilon=0.02$ ), the high-intensity gust response (for $\varepsilon=0.20$ ), and the GUST3D linear solution $[6,12]$.
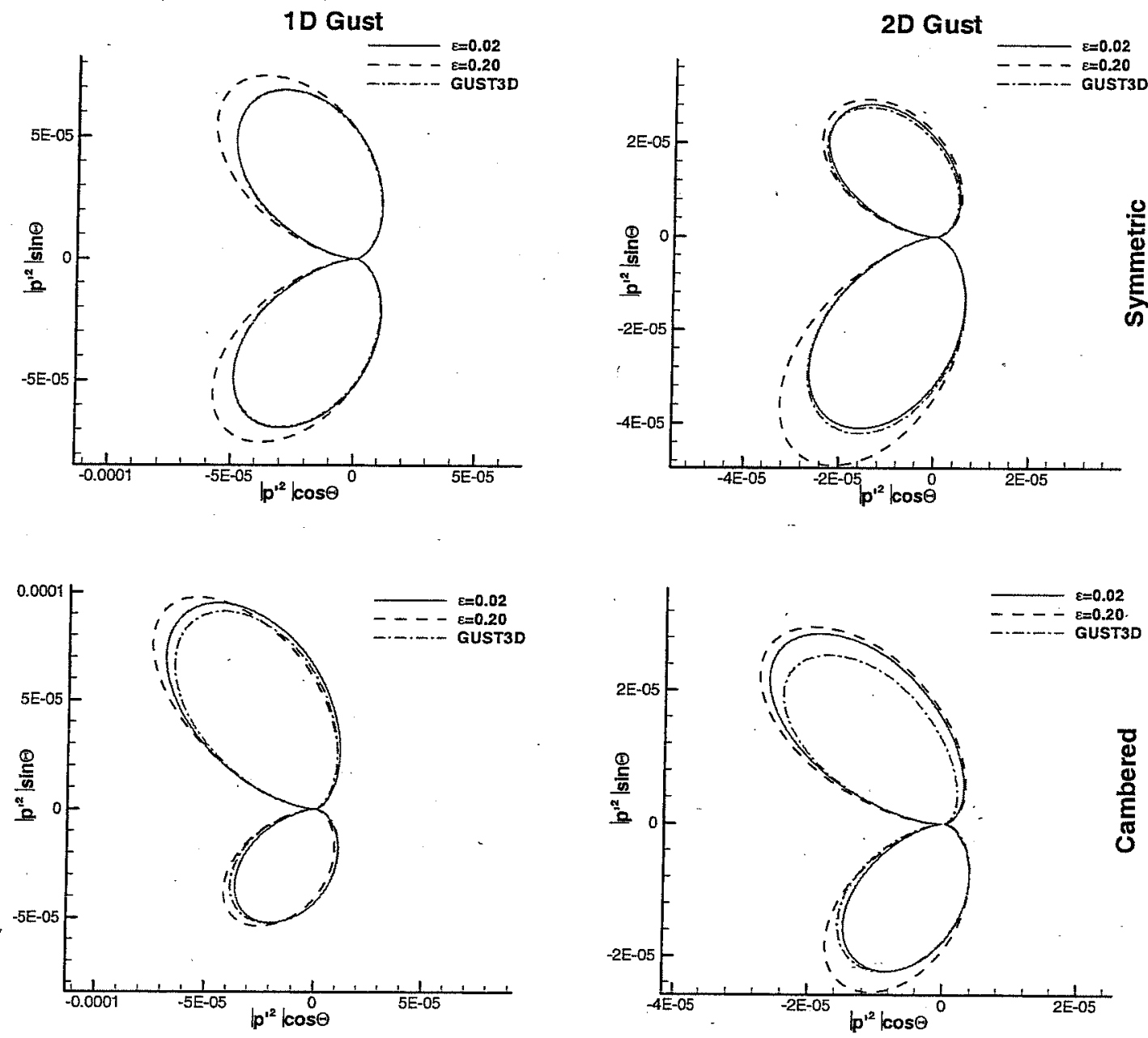

A detailed discussion of the high-intensity gust results is presented in the following paragraphs. The lowintensity solutions match well with GUST3D predictions, with only the case of high frequency and cambered profile showing apparent discrepancy (also in line with comparison in [4]).

In general, the comparison is better for the unloaded airfoil cases. It should be also mentioned that the unsteady pressure distribution on the airfoil shows an excellent match for all the considered cases, with results nearly identical to the previous comparison in [4].

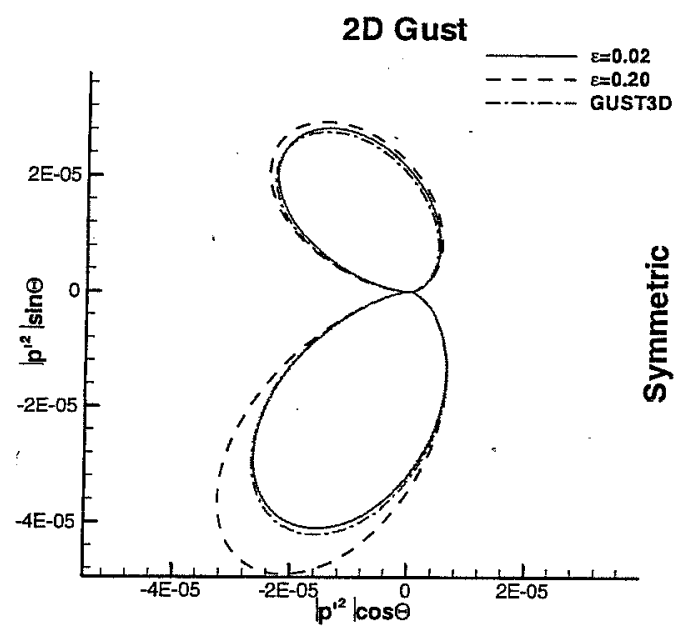

Figure 2:Distibution of $\left|\mathrm{p}^{\prime 2}\right|$ at $\mathrm{R}=1(\mathrm{k}=0.1)$

4

American Institute of Aeronautics and Astronautics 

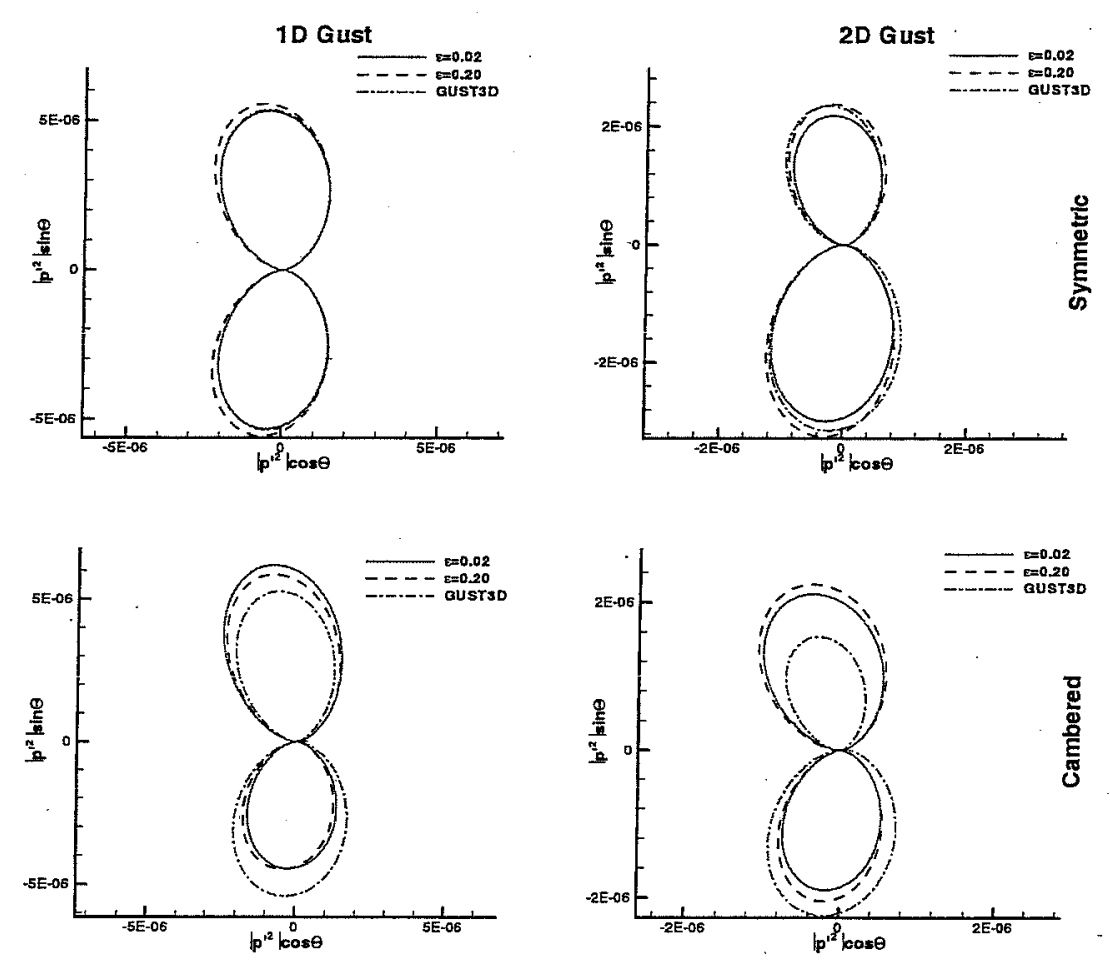

Figure 3:Disribution of $\left|\mathrm{p}^{2}\right|$ at $\mathrm{R}=4(\mathrm{k}=0.1)$
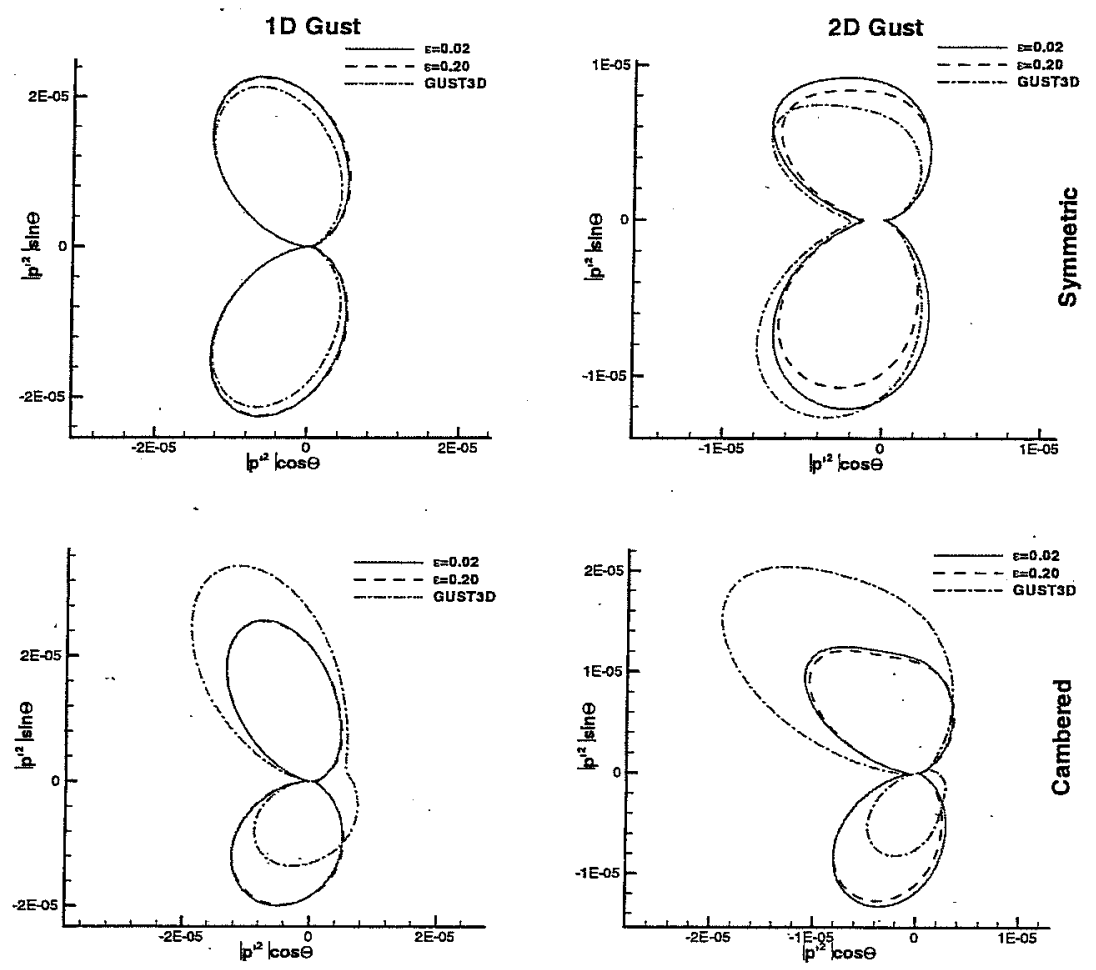

Figure 4:Distibution of $\left|\mathrm{p}^{, 2}\right|$ at $\mathrm{R}=1(\mathrm{k}=1.0)$

5

American Institute of Aeronautics and Astronautics 

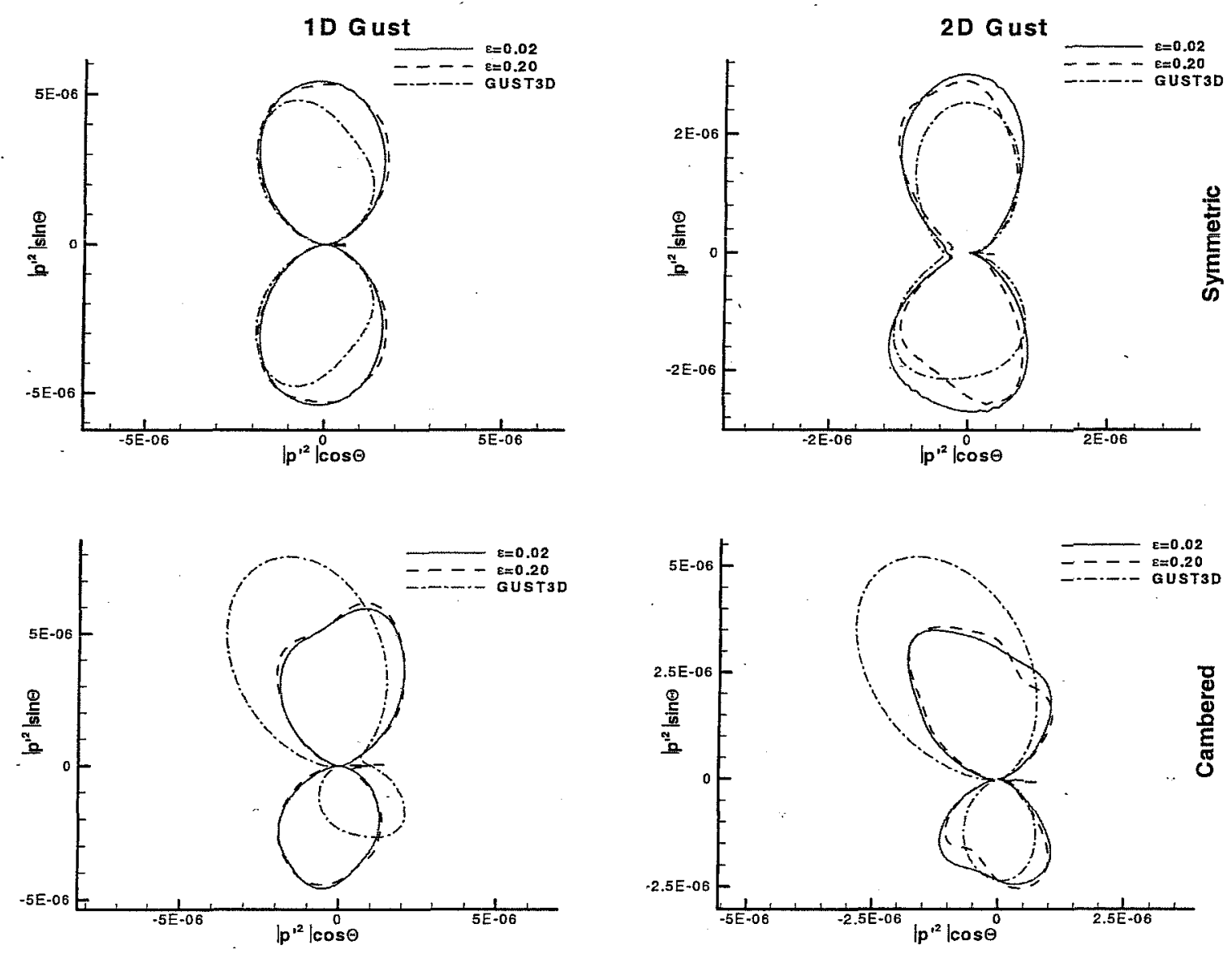

Figure 5:Disribution of $\left|\mathrm{p}^{2}\right|$ at $\mathrm{R}=4(\mathrm{k}=1.0)$

\section{Single-Frequency Gust}

Figures 2-5 also introduce a comparison between the solutions obtained for the low $(\varepsilon=0.02)$ and high $(\varepsilon=0.2)$ gust disturbance amplitudes. All the results in these and the following figures are scaled to the gust amplitude $\varepsilon=0.02$ for proper comparison. Note that at the low reduced frequency of $k=0.1$, the directivity patterns for all the cases retain the same shape, but, at least in the near field for $\mathrm{R}=1$, show a notable overall increase in the lobe acoustic intensity magnitudes, less evident in the far field $(R=4)$. For the higher reduced frequency of $\mathrm{k}=1.0$, the situation actually reverses, with only small lobe variation noticeable in the near field but rather significant change in the directivity shape in the far field, especially for the $2 \mathrm{D}$ gust cases. To examine this effect in more detail, Figure 6 shows the variation of the acoustic intensity in the far field over a range of $2 \mathrm{D}$ gust amplitudes for the loaded airfoil case.
Note that multiple lobes start to develop in the directivity pattern with increasing gust amplitude, which may point to the appearance of new non-compact sources in the domain. Indeed, the numerical analysis indicates that this phenomenon is connected to the development of the inviscid wake instability triggered by the breakdown of the gust at the airfoil surface (first noticed by Lockard and Morris [2] for the viscous case).

To illustrate this phenomenon, Figures 7-10 show contour plots for the instantaneous vertical velocity component. The results imply an increasing vorticity downstream in the wake, with the growth much more rapid for higher $\varepsilon$. 
Moreover, a similar trend is noted in Figure 11 for the RMS unsteady pressure fluctuations in the wake, which gives rise to the spike in the directivity pattern across the wake region, observed in Figure 6.

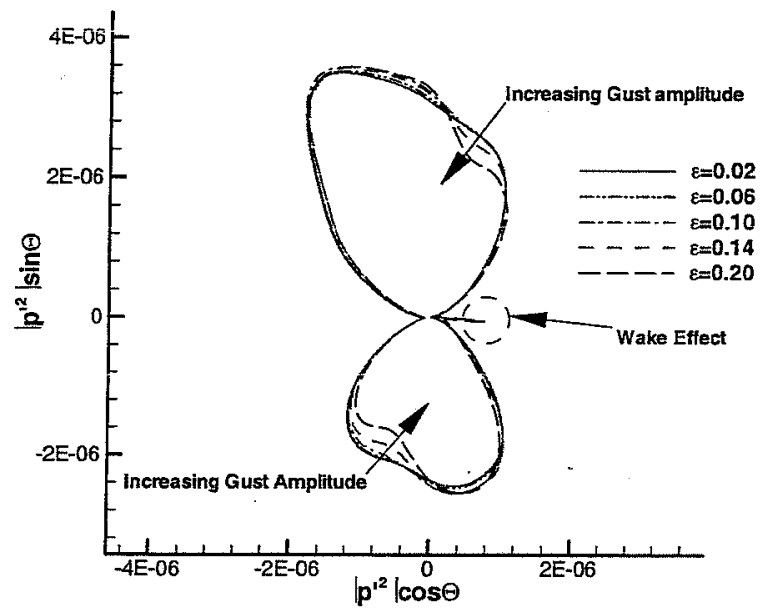

Figure 6: Effect of $\varepsilon$ on $\left|p^{\prime 2}\right|$ Disribution at $R=4(k=1.0$, $2 \mathrm{D}$ gust, cambered airfoil)

Thus, the lobe distortion in Figure 6 is apparently attributed to the wave-like instability development in the wake as the radiation pattern from such instability waves may develop a multi-lobe response (this phenomenon is examined in [13] using an integral Kirchhoff technique). Also, from Figures 3 and 5, one may notice that the $2 \mathrm{D}$ gust produces a more significant nonlinear effect on directivity, which may possibly be attributed to the fact that the longitudinal gust component induces additional shear in the wake thus contributing to instability development. All these hypotheses will be validated in a future analysis. Note that the near-field results $(R=1)$ do not reveal these directivity distortions since major nonlinear wake evolution occurs further downstream.

Figure 12 illustrates the contours of the calculated instantaneous unsteady pressure distribution in the domain. In order to examine the higher harmonics of the unsteady gust response, the FFT of the unsteady lift coefficient is carried out for all the test cases. Figure 13 shows the results scaled to the same input gust amplitude for comparison. In the analysis, the FFT is performed over a period corresponding to the nondimensional time of $40 \pi$. For this period, the code switches from an adjustable time step (satisfying the stability criteria) to the fixed one, using small steps to march through more than 30,000 iterations. It is obvious from Figure 13 that the level of higher harmonics is greatly increased at the higher gust intensity. A single case of unsteady response of the cambered airfoil to the ID gust with amplitude $\varepsilon=0.20$ also reveals harmonics of an unexplained spurious frequency.

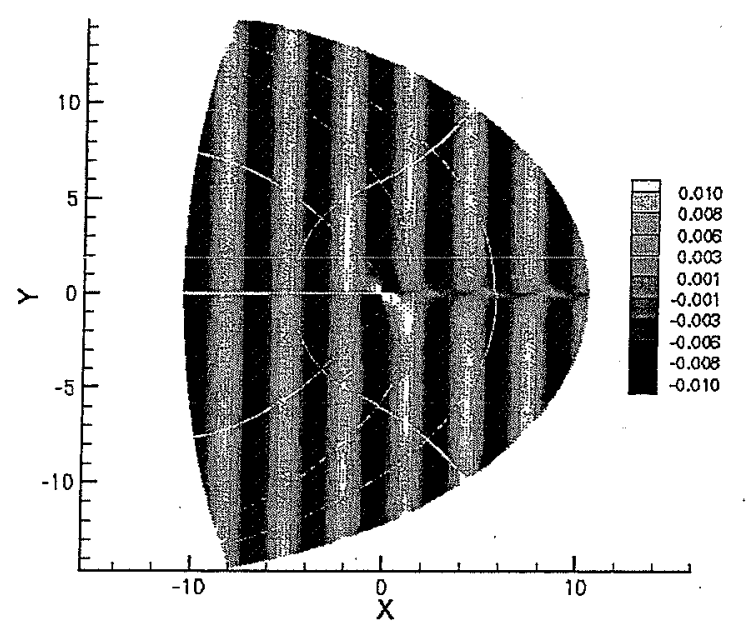

Figure 7: Instantaneous velocity, $\mathrm{Y}$-component: $\mathrm{k}=1.0$, 1D gust, $\varepsilon=0.02$, symmetric airfoil.

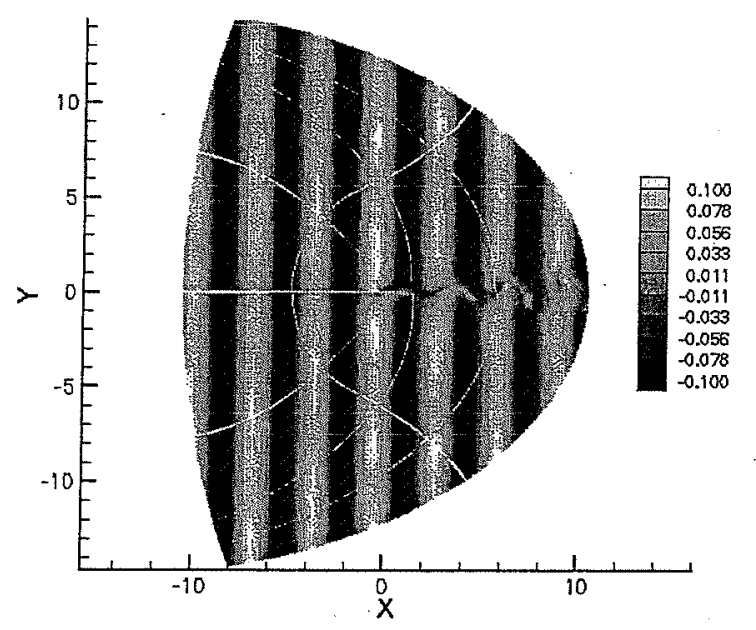

Figure 8: Instantaneous velocity, $\mathrm{Y}$-component: $\mathrm{k}=1.0$, $1 \mathrm{D}$ gust, $\varepsilon=0.20$, symmetric airfoil. 


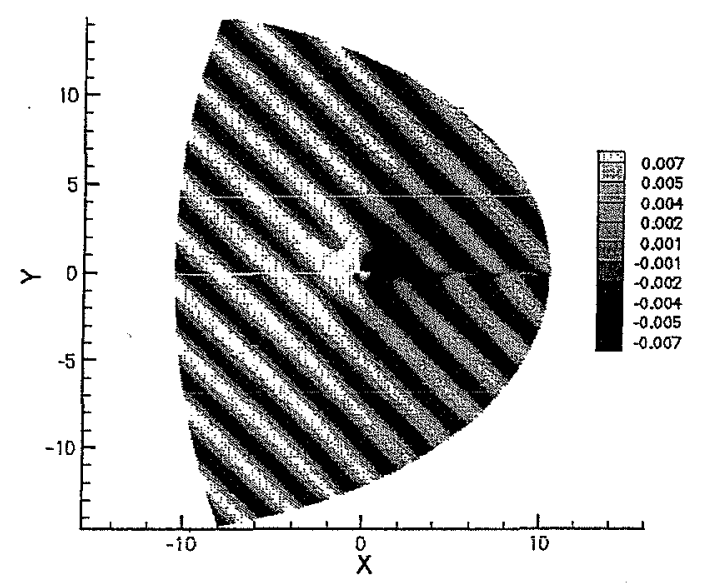

Figure 9: Instantaneous velocity, $Y$-component: $\mathrm{k}=1.0$, $2 \mathrm{D}$ gust, $\varepsilon=0.02$, cambered airfoil.

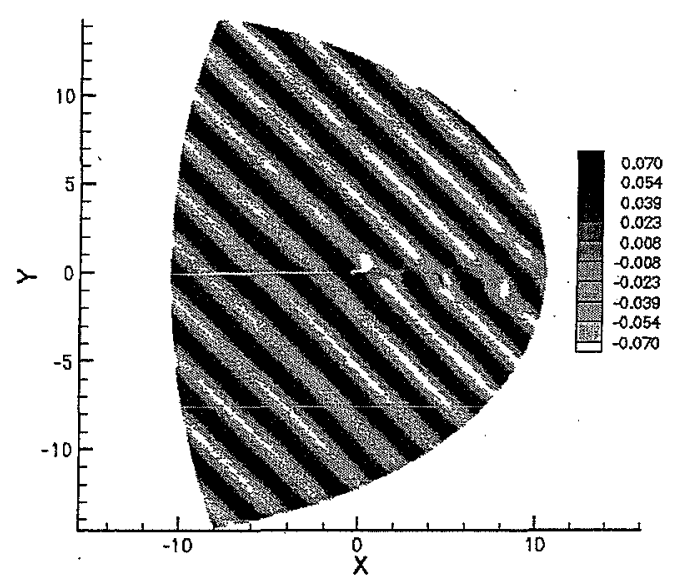

Figure 10: Instantaneous velocity, $\mathrm{Y}$-component: $\mathrm{k}=1.0$, $2 \mathrm{D}$ gust, $\varepsilon=0.20$, cambered airfoil.

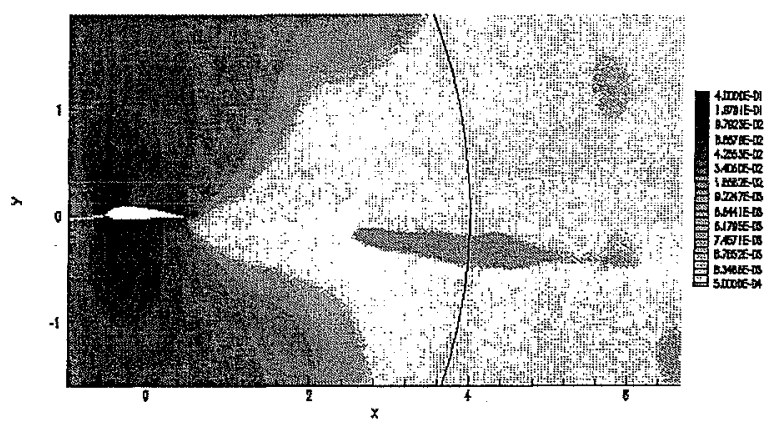

Figure 11: RMS unsteady pressure response contours downstream of airfoil (with $R=4$ line shown). $2 \mathrm{D}$ gust, $\mathrm{k}=1.0, \varepsilon=0.20$, cambered airfoil.

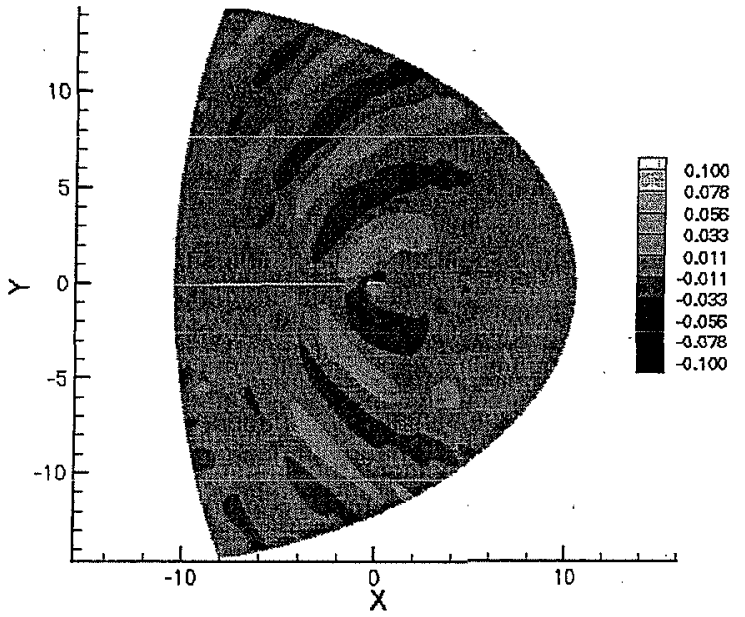

Figure 12: Contours of instantaneous unsteady pressure response. $2 \mathrm{D}$ gust, $\mathrm{k}=1.0, \varepsilon=0.20$, cambered airfoil.

It should be mentioned that for gust intensities higher than $\varepsilon=0.2$ and for higher gust frequencies, the nonlinear simulations start failing to converge as the spurious reflections start building up in the computational domain. This is attributed to the currently used formulation for the acoustic radiation boundary conditions based on the linear theory and the point source assumption. Future work will focus on resolving this issue to extend the analysis. This aspect is especially important because of the need to examine the nonlinear response at higher gust frequencies more typical of rotor/stator unsteady interactions.

\section{Multiple-Frequency Gust}

In order to further investigate the nonlinear response at high gust amplitudes, several simulations have been run with superposition of two different gust frequencies. Only the loaded airfoil is considered. In Figure 14, the FFT of the lift coefficient is presented for two superimposed $2 \mathrm{D}$ gust frequencies with $\mathrm{k}=0.6$ and $k=1.0$. The gusts are imposed with equal amplitudes, and comparison is presented for two cases with $\varepsilon=0.06$ and $\varepsilon=0.1$. As expected, the unsteady response reveals the presence of combination tones with $\mathrm{k}=0.4$ and $\mathrm{k}=1.6$, in addition to higher harmonics. All of them reveal higher amplitudes at higher gust intensities. Finally, for the same imposed gust amplitudes, Figure 15 compares the unsteady responses due to the superposition of ID vs. 2D gusts. Clearly, the superposition of $2 \mathrm{D}$ gusts generates higher levels of nonlinear response. 
$\varepsilon=0.02$
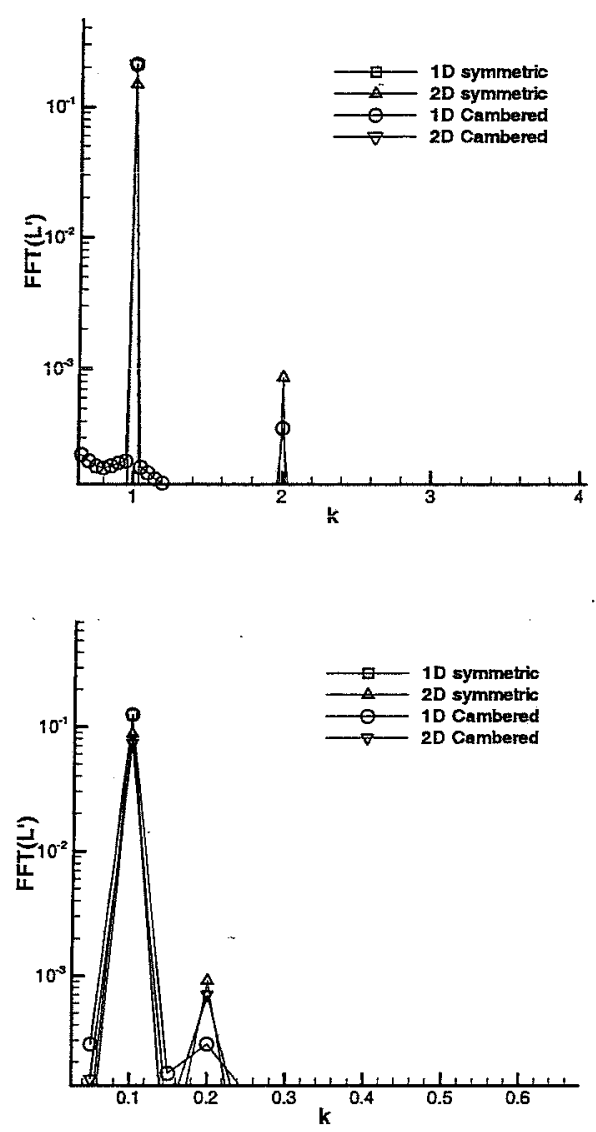

$\varepsilon=0.20$
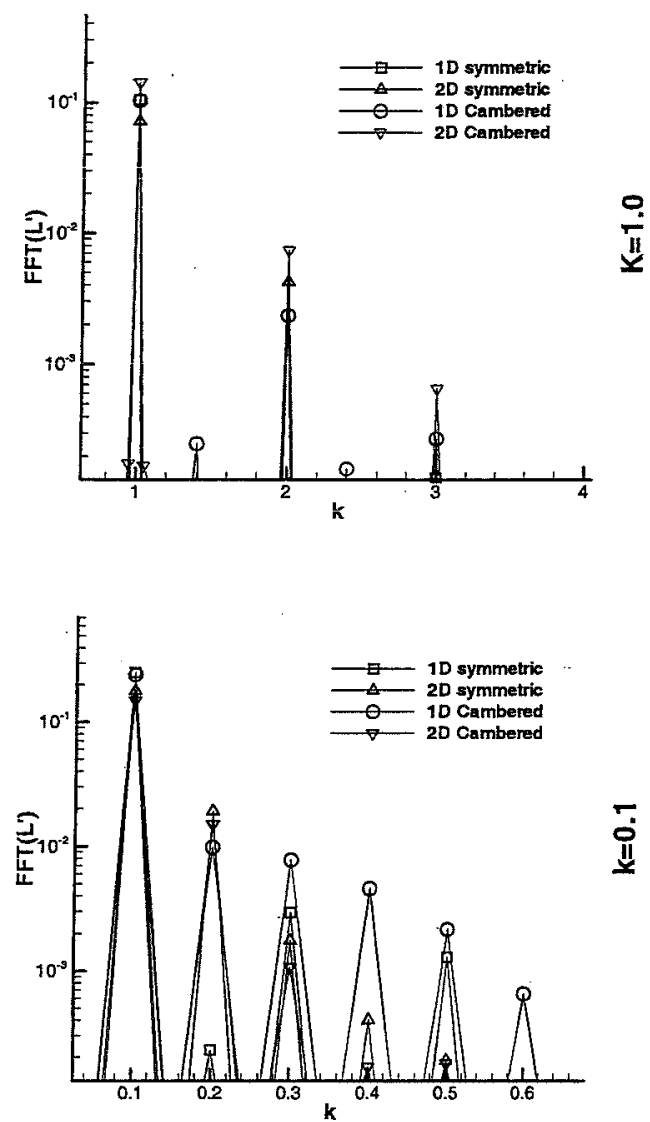

Figure13: FFT of the lift coefficient for the single imposed gust frequency.

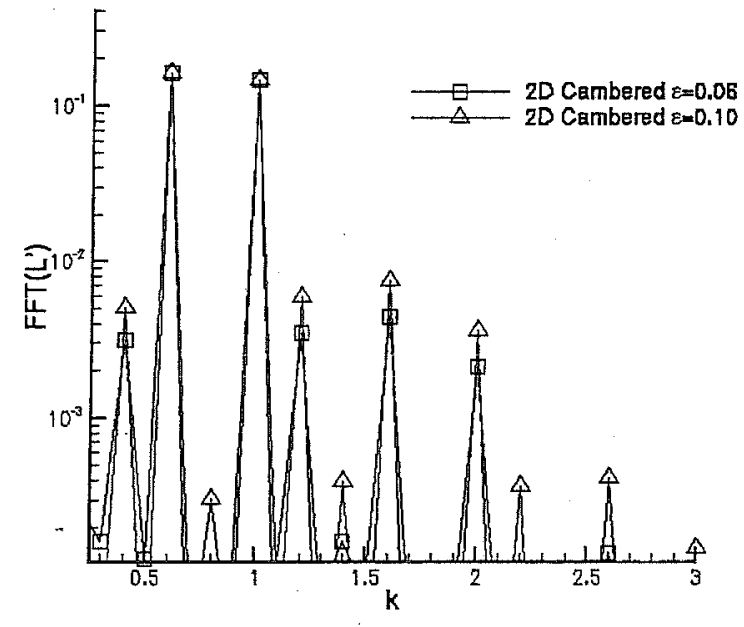

Figure 14: FFT of the lift coefficient for $\mathrm{k}=0.6+1.0$. Cambered airfoil, $2 \mathrm{D}$ gusts, cases with $\varepsilon=0.06$ and $\varepsilon=0.1$

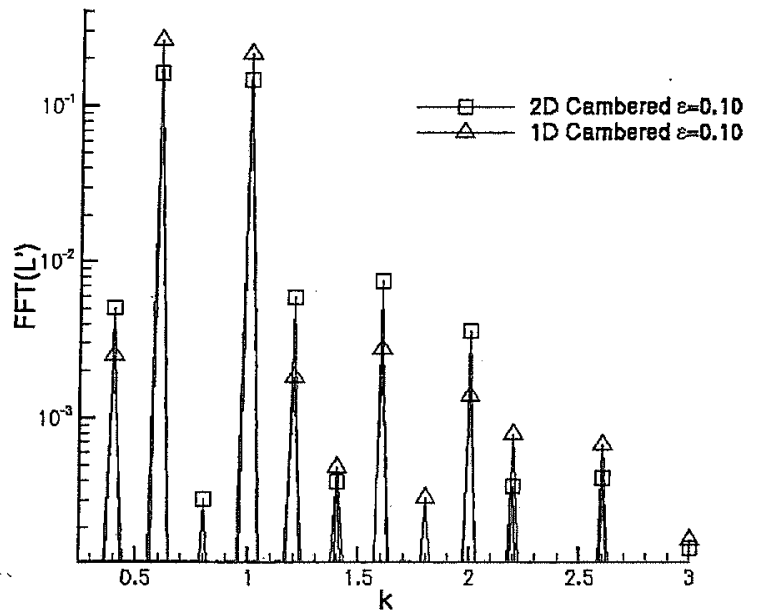

Figure 15: FFT of the lift coefficient for $\mathrm{k}=0.6+1.0$. Cambered airfoil, $1 \mathrm{D}$ and $2 \mathrm{D}$ gust cases, $\varepsilon=0.1$ 


\section{CONCLUSIONS}

This study focused on nonlinear effects of highintensity gust interaction with an airfoil. To investigate the limits of validity for the linearized approach, results of the nonlinear inviscid analysis were compared with the linearized Euler solutions in the parametric space of gust dimension, intensity, and frequency. Both loaded and unloaded airfoils in a subsonic flow were considered.

Among the nonlinear effects, generation of higher harmonics and combination tones associated with nonlinear self-interaction and exchange of energy between multiple imposed frequencies was observed for gust amplitudes approaching $20 \%$ of the mean flow. At low reduced frequency of $\mathrm{k}=0.1$, the double-lobe directivity patterns tended to retain its shape but increased the overall acoustic intensity magnitudes. On the other hand, for the higher reduced frequency of $\mathrm{k}=1.0$, most significant changes occurred in the directivity shapes starting to develop multiple lobes. This effect was attributed to the development of inviscid wake instability acting as a new non-compact source.

Overall, no significant deviation from the smallperturbation predictions was observed for gust amplitudes up to about $10 \%$ of the mean flow. It was further determined that, compared to the $1 D$ transverse gust, the $2 \mathrm{D}$ gust produced more significant nonlinear effects on the acoustic directivity in all cases considered, with the difference more pronounced at the higher reduced frequency. Nevertheless, no clear conclusion on the effects of gust frequency and airfoil loading on nonlinear response could be derived at this point. Future work is planned to extend this analysis to higher frequencies more typical of unsteady rotor-stator interactions, which will require development of a new approach to the acoustic boundary condition treatment more adequate for nonlinear computations. Effects of viscosity on nonlinear response particularly in the wake region will be examined with introduction of implicit time marching in the existing structure of the Very Large Eddy Simulation code used in this paper. The remaining discrepancies between the nonlinear analysis at low gust intensity and linear GUST3D solutions will be further investigated.

\section{ACKNOWLEDGEMENTS}

The authors would like to thank Rich Riendeau for his help and support in developing the Linux cluster for parallel computations. Dr. Golubev and Mr. Crivellini would like to acknowledge the support of the Ohio Aerospace Institute in this work.

\section{REFERENCES}

1. Verdon, J.M., "Review of Unsteady Aerodynamic Methods for Turbomachinery Aeroelastic and Aeroacoustics Applications," AIAA Journal, Vol. 31, pp. 235-250, 1993.

2. Lockard, D.P. and Morris, P.J., "Radiated Noise from Airfoils in Realistic Mean Flows," AIAA Journal, Vol.36, pp. 907-914, 1998.

3. Morse,P.M. and Ingard, K.U., "Theoretical Acoustics," Princeton Univ. Press, 1986.

4. Hixon, R., Mankbadi, R.R. and Scott, J.R., "Validation of a High-Order Prefactored Compact Code on Nonlinear Flows with Complex Geometries," AIAA Paper 2001-1103, Reno, NV, January 2001.

5. Hixon, R.R., Shih, S.-H., Mankbadi, R.R., and Scott, J.R., "Time Domain Solution of the Airfoil Gust Problem Using a High-Order Compact Scheme," AIAA Paper 98-3241, Cleveland, OH, 1998.

6. Scott, J.R. and Atassi, H.M., "A Finite-Difference, Frequency-Domain Numerical Scheme for the Solution of the Gust-Response Problem," Journal of Computational Physics, Vol. 119, pp.75-93, 1995.

7. Stanescu, D. and Habashi, W.G., "2N-Storage Low Dissipation and Dispersion Runge-Kutta Schemes for Computational Acoustics," Journal of Computational Physics, Vol.143, pp.674-681, 1998.

8. Hixon, R., "A New Class of Compact Schemes," AIAA Paper 98-0367, Reno, NV, January 1998.

9. GridPro ${ }^{\mathrm{TM}}$, Program Development Corporation, White Plains, NY.

10. Tam, C.K.W. and Webb, J.C., "DispersionRelation-Preserving Finite Difference Schemes for Computational Acoustics," Journal of Computational Physics, Vol. 107, pp.262-281, 1993.

11. Gropp, W., Lusk, E., Doss, N., and Skjellum, A., "A High-Performance, Portable Implementation of the MPI Message-Passing Interface Standard," Parallel Computing, Vol. 22(6), pp. 789-828, 1996.

12. Hariharan, S.I., Scott, J.R., and Kreider, K.L., "A Potential-Theoretic Method for Far-Field Sound Radiation Calculations," Journal of Computational Physics, Vol. 164, pp. 143-164, 2000.

13. Golubev, V.V., Mankbadi, R.R., and Dahl, M., "Prediction of the Acoustic Field Associated with Instability Wave Source Model for a Compressible Jet," accepted for presentation at the AIÄA/CEAS Aeroacoustics Conference, June 2002. 